\title{
Prevalence and Risk Factors of Postnatal Depression in Females with Cesarean Section and Normal Vaginal Delivery
}

\author{
Muhammad Asif', ${ }^{\star}$, Muhammad Saleem Rana' ${ }^{1}$,Asif Hanif', Syed Amir Gilani', Sultan Ayaz ${ }^{3}$, Ume Habiba ${ }^{2}$, \\ Benish Ali ${ }^{4}$ \\ 1 University Institute of Public Health, The University of Lahore, Pakistan. \\ ${ }^{2}$ Directorate of Medical Sciences, Department of Public Health, Government College University Faisalabad, \\ Pakistan. \\ ${ }^{3}$ Directorate of Medical Sciences, Department of Eastern Medicine, Government College University Faisalabad, \\ Pakistan. \\ ${ }^{4}$ Directorate of Medical Sciences, Department of Orthotics and prosthetics, Government College University \\ Faisalabad, Pakistan.
}

\author{
Authors' Contributions \\ 1 Conception \& Study design, Data Collection, \\ Data Analysis and /or interpretation. \\ 2 Conception \& Study design. \\ 3 Data Collection \& Processing. \\ 4 Data Analysis and /or interpretation. \\ 5 \& 6 Critical Review. \\ 7 Data Collection. \\ Acknowledgement \\ None \\ Article info. \\ Received: September 29, 2020 \\ Accepted: December 04, 2020 \\ Funding Source: Nil \\ Conflict of Interest: Nil \\ Cite this article: Asif $M$, Rana MS, Hanif A, \\ Gilani SA, Ayaz S, Habiba U, Ali B. Prevalence \\ and Risk Factors of Postnatal Depression in \\ Females with Cesarean Section and Normal \\ Vaginal Delivery. RADS J Pharm Pharm Sci. \\ 2020; 8(3):161-167.
}

*Address of Correspondence Author: muhammadasif@gcuf.edu.pk

\begin{abstract}
A BSTRACT
Objectives: To find the prevalence and risk factors of postnatal depression and association of parenting sense of competence with postnatal depression among females with cesarean section and normal vaginal delivery.

Methods: This case-control study was conducted in Allied hospital and Children hospital Faisalabad during a period of 1-1-2019 to 30-06-2020. A non-probability purposive sampling technique was used to enroll 284 women. The Urdu version of Edinburg Postnatal Depression Scale was used to measure postnatal depression.
\end{abstract}

Results: The mean age (in years) \pm SD was $27.39 \pm 5.26$ (min 18 years, max 45 years). According to cut-off score $\geq 13$ on EPDS $37.3 \%$ women were found depressed while $62.7 \%$ women were found non-depressed. The mean EPDS score \pm SD was $10.12 \pm 6.27$ (min score 0 ; max score 27 ). The women's age, women's education, education of head of family, monthly income of family and socio-economic status, mode of delivery, delivery place, number of pregnancies, history of infant death, history of child death, history of miscarriage and number of living children and parenting sense of competence were significantly associated with postnatal depression $(p<0.05)$.

Conclusion: Socio-demographic, obstetric risk factors, and parenting sense of competence are significant predictors of postnatal depression that need to be addressed in order to sustain safe motherhood.

Keywords: Postnatal Depression, Risk factors, EPDS, Parenting knowledge.

\section{INTRODUCTION}

Postpartum depression (PND) is currently an important public health problem due to its significant adverse effects on mothers and babies. In recent years, the prevalence of postpartum depression (PND) has been increasing, and it has become one of the most important public health problems. According to an estimate, nearly $10-15 \%$ of puerperal women are suffering from postnatal depression worldwide [1]. In high-income countries, the prevalence ranges from $10 \%$ to $15 \%$. However, in low- and middle-income countries, the rate varies from $16 \%$ to $36 \%$ [2]. In Asian countries, the prevalence of postnatal 
depression has been stated as between $0.5 \%$ to $60 \%$ [3] and between $3.5 \%$ to $63.3 \%$ [4]. Some of the risk factors include obstetrical history, biological factors, lifestyle, stressful life events, previous history of clinical depression and emotional support [5]. The high prevalence of postnatal depression in women signifies an important public health concern that is needed to address in order to improve the health of mother and child. Early identification and intervention is crucial for eliminating the adverse effects on child development and ensuring safe motherhood $[3,6,7]$. The Edinburgh Postpartum Depression Scale (EPDS), previously developed in the UK, is one of the most commonly used screening tools for measuring symptoms of depression and anxiety disorders in the perinatal period (PCMD) [8].

Since the prevalence of postpartum depression in Pakistan is very high according to the previous literature, it is necessary to evaluate the prevalence of postpartum depression and its related sociodemographic, economic and obstetric risk factors which can be modified in order to ensure better health of mother and child. This study aimed to find the prevalence and risk factors of postnatal depression and association of parenting sense of competence with postnatal depression among females with cesarean section and normal vaginal delivery.

\section{METHODS}

This Case-control study was conducted in Allied hospital and Children hospital Faisalabad during a period of 18 months (1st Jan 2019 to 30 June 2020). By using non-probability purposive sampling technique 284 puerperal women (142 with normal vaginal delivery and 142 with cesarean section) were included in the study. The sample size was calculated using $90 \%$ power of test, $95 \%$ confidence level, $5 \%$ margin of error, anticipated depression in females with normal vaginal delivery as $13.6 \%$ [9] and anticipated depression in females with C-section as $27.6 \%$ [9].

Women aged 18-45 years, who had recently given birth either by cesarean section or vaginal delivery were included in the study. Women who needed emergency care for any medical or psychiatric problem and women with neonatal deaths and fetal anomalies were not included in the study.
The Edinburgh Postpartum Depression Scale is used to measure postpartum depression. The questionnaire includes 10 questions based on the

four-point Likert scale. A score of 13 or more indicates postpartum depression[10]. Data collecting tool was a self-administered questionnaire translated into Urdu that contained variables related to sociodemographic and obstetric history of puerperal women and parenting sense of competence scale to measure the association of child raising skills/knowledge and parenting comfort with postnatal depression.

When conducting research, follow the rules and regulations established by the Ethics Committee of Lahore University and respect the rights of research participants.

\section{Data Analysis:}

Used SPSS 23 to analyze the data. Continuous data are expressed as mean \pm SD. Nominal data is expressed as frequency and percentage. Chi-square independence test is used to measure the relationship between sociodemographic and obstetric risk factors and postpartum depression. Chi-square independence test is also used to measure the relationship between parental awareness of ability and postpartum depression. A $p$ value $<0.05$ is considered to show a statistically significant result.

\section{RESULTS}

A total of 284 puerperal women were included in the study. The mean age (in years) \pm SD was $27.39 \pm$ 5.26. The minimum age was 18 years and maximum age was 45 years. According to cut-off score $\geq 13$ on Edinburg postnatal depression scale 106 (37.3\%) women were found depressed while 178 (62.7\%) women were found non-depressed. The mean EPDS score \pm SD was $10.12 \pm 6.27$ ( $\min$ score 0 ; max score 27).

The chi-square test of independence revealed that the women's age, women's education, education of head of family, monthly income of family and socioeconomic status were significantly associated with postnatal depression $(p<0.05)$, whereas the occupation of head of family had no significant association with postnatal depression among puerperal women $(p>0.05)$. (Table 1)

The chi-square test of independence revealed that the mode of delivery, delivery place, number of 
pregnancies, history of infant death, history of child death, history of miscarriage and number of living children were significantly associated with postnatal depression $(p<0.05)$, however, the contraception had no significant effect on postnatal depression ( $p>0.05)$. (Table 2)

The association of parenting skills/knowledge and parenting valuing/comfort with postnatal depression is elaborated in Table $\mathbf{3}$.

Table 1. Chi-Square test of Independence to Measure Association of Socio-Demographic Variables with Postnatal Depression.

\begin{tabular}{|c|c|c|c|c|c|c|c|}
\hline \multirow{2}{*}{$\begin{array}{l}\text { Socio- } \\
\text { Demographic } \\
\text { Risk Factors }\end{array}$} & \multirow{2}{*}{ Responses } & \multirow{2}{*}{$\mathbf{N}(\%)$} & \multicolumn{2}{|c|}{ EPDS Categories } & \multirow{2}{*}{$\begin{array}{c}\% \text { of } \\
\text { Depression }\end{array}$} & \multirow{2}{*}{$\begin{array}{l}X^{2} \\
(d f)\end{array}$} & \multirow{2}{*}{$\begin{array}{c}\mathrm{p}- \\
\text { value }\end{array}$} \\
\hline & & & $\begin{array}{c}\text { Non- } \\
\text { Depressed }\end{array}$ & Depressed & & & \\
\hline $\begin{array}{l}\text { Age Groups } \\
\text { (in years) }\end{array}$ & $\begin{array}{l}18-24 \\
25-31 \\
32-38 \\
39-45\end{array}$ & $\begin{array}{c}79(27.8) \\
150(52.8) \\
48(16.9) \\
7(2.5)\end{array}$ & $\begin{array}{c}42 \\
101 \\
35 \\
0\end{array}$ & $\begin{array}{c}37 \\
49 \\
13 \\
7\end{array}$ & $\begin{array}{c}46.8 \\
32.7 \\
27.1 \\
100.0\end{array}$ & $\begin{array}{c}18.35 \\
(3)\end{array}$ & $<0.001$ \\
\hline $\begin{array}{l}\text { Women's } \\
\text { Education }\end{array}$ & $\begin{array}{c}\text { Illiterate } \\
\text { Primary } \\
\text { Middle } \\
\text { Matric } \\
\text { Intermediate } \\
\text { Post Graduate } \\
\text { Professional } \\
\text { Degree }\end{array}$ & $\begin{array}{c}22(7.7) \\
25(8.8) \\
38(13.4) \\
79(27.8) \\
53(18.7) \\
40(14.1) \\
27(9.5)\end{array}$ & $\begin{array}{l}15 \\
14 \\
18 \\
43 \\
42 \\
29 \\
17\end{array}$ & $\begin{array}{c}7 \\
11 \\
20 \\
36 \\
11 \\
11 \\
10\end{array}$ & $\begin{array}{l}31.8 \\
44.0 \\
52.6 \\
45.6 \\
20.8 \\
27.5 \\
37.0\end{array}$ & $\begin{array}{c}14.73 \\
(6)\end{array}$ & 0.02 \\
\hline $\begin{array}{l}\text { Education of } \\
\text { Head of Family }\end{array}$ & $\begin{array}{c}\text { Illiterate } \\
\text { Primary } \\
\text { Middle } \\
\text { Matric } \\
\text { Intermediate } \\
\text { Post Graduate } \\
\text { Professional } \\
\text { Degree }\end{array}$ & $\begin{array}{c}18(6.3) \\
18(6.3) \\
26(9.2) \\
78(27.5) \\
58(20.4) \\
54(19.0) \\
32(11.3)\end{array}$ & $\begin{array}{l}13 \\
11 \\
20 \\
36 \\
44 \\
34 \\
20\end{array}$ & $\begin{array}{c}5 \\
7 \\
6 \\
42 \\
14 \\
20 \\
12\end{array}$ & $\begin{array}{l}27.8 \\
38.9 \\
23.1 \\
53.8 \\
24.1 \\
37.0 \\
37.5\end{array}$ & $\begin{array}{c}16.39 \\
(6)\end{array}$ & 0.01 \\
\hline $\begin{array}{l}\text { Occupation of } \\
\text { Head of Family }\end{array}$ & $\begin{array}{l}\text { Professional } \\
\text { Semi- } \\
\text { Professional } \\
\text { Clerical } \\
\text { Skilled Worker } \\
\text { Semi-Skilled }\end{array}$ & $\begin{array}{c}21(7.4) \\
103(36.3) \\
56(19.7) \\
73(25.7) \\
31(10.9)\end{array}$ & $\begin{array}{l}14 \\
62 \\
39 \\
43 \\
20\end{array}$ & $\begin{array}{c}7 \\
41 \\
17 \\
30 \\
11\end{array}$ & $\begin{array}{l}33.3 \\
39.8 \\
30.4 \\
41.1 \\
35.5\end{array}$ & $\begin{array}{c}2.06 \\
(4)\end{array}$ & 0.72 \\
\hline $\begin{array}{l}\text { Monthly } \\
\text { Income of } \\
\text { Family }\end{array}$ & $\begin{array}{c}\geq 52,734 \\
26,355-52,733 \\
19,759-26,354 \\
13,161-19,758 \\
7,887-13,160 \\
2,641-7,886 \\
\leq 2,640\end{array}$ & $\begin{array}{l}64(22.5) \\
57(20.1) \\
61(21.5) \\
49(17.3) \\
21(7.4) \\
21(7.4) \\
11(3.9) \\
\end{array}$ & $\begin{array}{c}48 \\
43 \\
34 \\
25 \\
12 \\
10 \\
6\end{array}$ & $\begin{array}{c}16 \\
14 \\
27 \\
24 \\
9 \\
11 \\
5\end{array}$ & $\begin{array}{l}25.0 \\
24.6 \\
44.3 \\
49.0 \\
42.9 \\
52.4 \\
45.5 \\
\end{array}$ & $\begin{array}{c}14.85 \\
(6)\end{array}$ & 0.02 \\
\hline $\begin{array}{c}\text { Socio- } \\
\text { economic } \\
\text { Status on } \\
\text { Kuppuswamy's } \\
\text { Scale } \\
\end{array}$ & $\begin{array}{c}\text { Upper } \\
\text { Upper Middle } \\
\text { Lower Middle } \\
\text { Upper Lower }\end{array}$ & $\begin{array}{c}18(6.3) \\
119(41.9) \\
107(37.7) \\
40(14.1) \\
\end{array}$ & $\begin{array}{l}13 \\
84 \\
55 \\
26\end{array}$ & $\begin{array}{c}5 \\
35 \\
52 \\
14\end{array}$ & $\begin{array}{l}27.8 \\
29.4 \\
48.6 \\
35.0 \\
\end{array}$ & $\begin{array}{l}9.79 \\
(3)\end{array}$ & 0.02 \\
\hline
\end{tabular}


Table 2. Chi-Square test of Independence to Measure Association of Obstetric Risk Factors with Postnatal Depression.

\begin{tabular}{|c|c|c|c|c|c|c|c|}
\hline \multirow{2}{*}{$\begin{array}{c}\text { Obstetric } \\
\text { Risk Factors }\end{array}$} & \multirow{2}{*}{ Responses } & \multirow{2}{*}{ N (\%) } & \multicolumn{2}{|c|}{ EPDS Categories } & \multirow{2}{*}{$\begin{array}{c}\% \text { of } \\
\text { Depression }\end{array}$} & \multirow{2}{*}{$\begin{array}{l}X^{2} \\
\text { (df) }\end{array}$} & \multirow{2}{*}{$\begin{array}{c}\text { p- } \\
\text { value }\end{array}$} \\
\hline & & & $\begin{array}{c}\text { Non- } \\
\text { Depressed }\end{array}$ & Depressed & & & \\
\hline \multirow{2}{*}{$\begin{array}{l}\text { Mode of } \\
\text { Delivery }\end{array}$} & Vaginal Delivery & $142(50.0)$ & 101 & 41 & 38.7 & 8.67 & \multirow{2}{*}{0.003} \\
\hline & Cesarean Section & $142(50.0)$ & 77 & 65 & 61.3 & (1) & \\
\hline \multirow{6}{*}{$\begin{array}{l}\text { Newborn } \\
\text { Child's Age } \\
\text { (in months) }\end{array}$} & 1 & $105(37.0)$ & 78 & 27 & 25.7 & \multirow{6}{*}{$\begin{array}{c}18.19 \\
(5)\end{array}$} & \multirow{6}{*}{0.003} \\
\hline & 2 & 39 (13.7) & 24 & 15 & 38.5 & & \\
\hline & 3 & $18(6.3)$ & 6 & 12 & 66.7 & & \\
\hline & 4 & $23(8.1)$ & 9 & 14 & 60.9 & & \\
\hline & 5 & $21(7.4)$ & 13 & 8 & 38.1 & & \\
\hline & 6 & $78(27.5)$ & 48 & 30 & 38.5 & & \\
\hline \multirow{3}{*}{$\begin{array}{l}\text { Delivery } \\
\text { Place }\end{array}$} & Home & $56(19.7)$ & 38 & 18 & 32.1 & \multirow{3}{*}{$\begin{array}{c}12.06 \\
(2)\end{array}$} & \multirow{3}{*}{0.002} \\
\hline & Hospital & $211(74.3)$ & 136 & 75 & 35.5 & & \\
\hline & Other & $17(6.0)$ & 4 & 13 & 76.5 & & \\
\hline \multirow{4}{*}{$\begin{array}{l}\text { Number of } \\
\text { Pregnancies }\end{array}$} & 1 & $62(21.8)$ & 53 & 9 & 14.5 & & \multirow{4}{*}{$<0.001$} \\
\hline & 2 & $91(32.0)$ & 59 & 32 & 35.2 & 24.04 & \\
\hline & 3 & $58(20.4)$ & 30 & 28 & 48.3 & (3) & \\
\hline & $\geq 4$ & $70(24.6)$ & 33 & 37 & 52.9 & & \\
\hline \multirow{2}{*}{$\begin{array}{l}\text { Infant Death } \\
\text { (under } 1 \text { year) }\end{array}$} & Yes & $53(18.7)$ & 23 & 30 & 56.6 & 10.35 & \multirow{2}{*}{0.001} \\
\hline & No & $231(81.3)$ & 155 & 76 & 32.9 & (1) & \\
\hline \multirow{2}{*}{$\begin{array}{l}\text { Child Death } \\
\text { (under } 5 \\
\text { years) }\end{array}$} & Yes & $36(12.7)$ & 6 & 30 & 83.3 & 37.31 & \multirow{2}{*}{$<0.001$} \\
\hline & No & $248(87.3)$ & 172 & 76 & 30.6 & (1) & \\
\hline \multirow{2}{*}{$\begin{array}{l}\text { History of } \\
\text { Miscarriage }\end{array}$} & Yes & $71(25.0)$ & 31 & 40 & 56.3 & 14.63 & \multirow{2}{*}{$<0.001$} \\
\hline & No & $213(75.0)$ & 147 & 66 & 31.0 & (1) & \\
\hline \multirow{4}{*}{$\begin{array}{l}\text { Number of } \\
\text { Living } \\
\text { Children }\end{array}$} & 1 & $94(33.1)$ & 72 & 22 & 23.4 & & \multirow{4}{*}{0.004} \\
\hline & 2 & 99 (34.9) & 51 & 48 & 48.5 & 13.34 & \\
\hline & 3 & 67 (23.6) & 41 & 26 & 38.8 & (3) & \\
\hline & $\geq 4$ & $24(8.5)$ & 14 & 10 & 41.7 & & \\
\hline \multirow{2}{*}{ Contraception } & Yes & $42(14.8)$ & 29 & 13 & 31.0 & 0.85 & \multirow{2}{*}{0.35} \\
\hline & No & $242(85.2)$ & 149 & 93 & 38.4 & (1) & \\
\hline
\end{tabular}


Table 3. Chi-Square test of Independence to Measure Association of Parenting Sense of Competence With Postnatal Depression.

\begin{tabular}{|c|c|c|c|c|c|c|}
\hline \multirow[b]{2}{*}{ Parenting Sense of Competence Scale } & \multirow[b]{2}{*}{ Response } & \multicolumn{2}{|c|}{ EPDS Categories } & \multirow{2}{*}{$\begin{array}{c}\% \text { of } \\
\text { Depression }\end{array}$} & \multirow{2}{*}{$\begin{array}{l}X^{2} \\
(d f)\end{array}$} & \multirow{2}{*}{$\begin{array}{c}\mathrm{p}- \\
\text { value }\end{array}$} \\
\hline & & $\begin{array}{c}\text { Non- } \\
\text { Depressed }\end{array}$ & Depressed & & & \\
\hline \multicolumn{7}{|l|}{ (Skills/Knowledge Sub-scale questions) } \\
\hline $\begin{array}{l}\text { The problems of taking care of a child are } \\
\text { easy to solve once you know how your } \\
\text { actions affect your child, an understanding I } \\
\text { have acquired. }\end{array}$ & $\begin{array}{l}\text { Agree } \\
\text { Disagree }\end{array}$ & $\begin{array}{c}169 \\
9\end{array}$ & $\begin{array}{l}89 \\
17\end{array}$ & $\begin{array}{l}34.5 \\
65.4\end{array}$ & $\begin{array}{l}9.63 \\
(1)\end{array}$ & 0.002 \\
\hline $\begin{array}{l}\text { I would make a fine model for a new mother } \\
\text { to follow in order to learn what she would } \\
\text { need to know in order to be a good parent. }\end{array}$ & $\begin{array}{l}\text { Agree } \\
\text { Disagree }\end{array}$ & $\begin{array}{c}171 \\
7\end{array}$ & $\begin{array}{l}80 \\
26\end{array}$ & $\begin{array}{l}31.9 \\
78.8\end{array}$ & $\begin{array}{l}27.44 \\
(1)\end{array}$ & $<0.001$ \\
\hline $\begin{array}{l}\text { Being a parent is manageable, and any } \\
\text { problems are easily solved. }\end{array}$ & $\begin{array}{c}\text { Agree } \\
\text { Disagree }\end{array}$ & $\begin{array}{c}164 \\
14\end{array}$ & $\begin{array}{l}73 \\
33\end{array}$ & $\begin{array}{l}30.8 \\
70.2\end{array}$ & $\begin{array}{l}26.04 \\
(1)\end{array}$ & $<0.001$ \\
\hline $\begin{array}{l}\text { A difficult problem in being a parent is not } \\
\text { knowing whether you're doing a good job or a } \\
\text { bad one }\end{array}$ & $\begin{array}{l}\text { Agree } \\
\text { Disagree }\end{array}$ & $\begin{array}{c}112 \\
66\end{array}$ & $\begin{array}{l}81 \\
25\end{array}$ & $\begin{array}{l}42.0 \\
27.5\end{array}$ & $\begin{array}{c}5.55 \\
(1)\end{array}$ & 0.02 \\
\hline $\begin{array}{l}\text { I meet my own personal expectations for } \\
\text { expertise in caring for my child. }\end{array}$ & $\begin{array}{c}\text { Agree } \\
\text { Disagree }\end{array}$ & $\begin{array}{c}162 \\
16\end{array}$ & $\begin{array}{l}63 \\
43\end{array}$ & $\begin{array}{l}28.0 \\
72.9\end{array}$ & $\begin{array}{l}40.25 \\
(1)\end{array}$ & $<0.001$ \\
\hline $\begin{array}{l}\text { If anyone can find the answer to what is } \\
\text { troubling my child, I am the one. }\end{array}$ & $\begin{array}{c}\text { Agree } \\
\text { Disagree }\end{array}$ & $\begin{array}{c}168 \\
10\end{array}$ & $\begin{array}{l}51 \\
55\end{array}$ & $\begin{array}{l}23.3 \\
84.6\end{array}$ & $\begin{array}{l}80.58 \\
(1)\end{array}$ & $<0.001$ \\
\hline $\begin{array}{l}\text { Considering how long I've been a mother, I } \\
\text { feel thoroughly familiar with this role. }\end{array}$ & $\begin{array}{c}\text { Agree } \\
\text { Disagree }\end{array}$ & $\begin{array}{c}161 \\
17\end{array}$ & $\begin{array}{l}68 \\
38\end{array}$ & $\begin{array}{l}29.7 \\
69.1\end{array}$ & $\begin{array}{l}29.42 \\
(1)\end{array}$ & $<0.001$ \\
\hline $\begin{array}{l}\text { I honestly believe I have all the skills } \\
\text { necessary to be a good mother to my child. }\end{array}$ & $\begin{array}{c}\text { Agree } \\
\text { Disagree }\end{array}$ & $\begin{array}{c}159 \\
19\end{array}$ & $\begin{array}{l}68 \\
38\end{array}$ & $\begin{array}{l}30.0 \\
66.7\end{array}$ & $\begin{array}{c}26.24 \\
(1)\end{array}$ & $<0.001$ \\
\hline \multicolumn{7}{|l|}{ (Valuing/Comfort Sub-scale questions) } \\
\hline $\begin{array}{l}\text { Even though being a parent could be } \\
\text { rewarding, I am frustrated now while my child } \\
\text { is at his/her present age. }\end{array}$ & $\begin{array}{l}\text { Agree } \\
\text { Disagree }\end{array}$ & $\begin{array}{c}114 \\
64\end{array}$ & $\begin{array}{l}91 \\
15\end{array}$ & $\begin{array}{l}44.4 \\
19.0\end{array}$ & $\begin{array}{l}15.73 \\
(1)\end{array}$ & $<0.001$ \\
\hline $\begin{array}{l}\text { I go to bed the same way I wake up in the } \\
\text { morning, feeling I have not accomplished a } \\
\text { whole lot. }\end{array}$ & $\begin{array}{l}\text { Agree } \\
\text { Disagree }\end{array}$ & $\begin{array}{c}116 \\
62\end{array}$ & $\begin{array}{l}88 \\
18\end{array}$ & $\begin{array}{l}43.1 \\
22.5\end{array}$ & $\begin{array}{c}10.46 \\
(1)\end{array}$ & 0.001 \\
\hline $\begin{array}{l}\text { I do not know why it is, but sometimes when } \\
\text { I'm supposed to be in control, I feel more like } \\
\text { the one being manipulated. }\end{array}$ & $\begin{array}{l}\text { Agree } \\
\text { Disagree }\end{array}$ & $\begin{array}{c}112 \\
66\end{array}$ & $\begin{array}{l}93 \\
13\end{array}$ & $\begin{array}{l}45.4 \\
16.5\end{array}$ & $\begin{array}{l}20.37 \\
(1)\end{array}$ & $<0.001$ \\
\hline $\begin{array}{l}\text { My mother was better prepared to be a good } \\
\text { mother than I am. }\end{array}$ & $\begin{array}{l}\text { Agree } \\
\text { Disagree }\end{array}$ & $\begin{array}{l}151 \\
27\end{array}$ & $\begin{array}{l}87 \\
19\end{array}$ & $\begin{array}{l}36.6 \\
41.3\end{array}$ & $\begin{array}{l}0.37 \\
(1)\end{array}$ & 0.54 \\
\hline $\begin{array}{l}\text { Sometimes I feel like I'm not getting anything } \\
\text { done. }\end{array}$ & $\begin{array}{l}\text { Agree } \\
\text { Disagree }\end{array}$ & $\begin{array}{c}106 \\
72\end{array}$ & $\begin{array}{l}83 \\
23\end{array}$ & $\begin{array}{l}43.9 \\
24.2\end{array}$ & $\begin{array}{l}10.49 \\
(1)\end{array}$ & 0.001 \\
\hline $\begin{array}{l}\text { My talents and interests are in other areas, } \\
\text { not being a parent. }\end{array}$ & $\begin{array}{l}\text { Agree } \\
\text { Disagree }\end{array}$ & $\begin{array}{c}103 \\
75\end{array}$ & $\begin{array}{l}56 \\
50\end{array}$ & $\begin{array}{l}35.2 \\
40.0\end{array}$ & $\begin{array}{c}0.68 \\
(1)\end{array}$ & 0.41 \\
\hline $\begin{array}{l}\text { If being a mother of a child were only more } \\
\text { interesting, I would be motivated to do a } \\
\text { better job as a parent. }\end{array}$ & $\begin{array}{l}\text { Agree } \\
\text { Disagree }\end{array}$ & $\begin{array}{c}133 \\
45\end{array}$ & $\begin{array}{l}86 \\
20\end{array}$ & $\begin{array}{l}39.3 \\
30.8\end{array}$ & $\begin{array}{c}1.54 \\
(1)\end{array}$ & 0.21 \\
\hline Being a parent makes me tense and anxious. & $\begin{array}{c}\text { Agree } \\
\text { Disagree }\end{array}$ & $\begin{array}{l}151 \\
27\end{array}$ & $\begin{array}{l}86 \\
20\end{array}$ & $\begin{array}{l}36.3 \\
42.6\end{array}$ & $\begin{array}{c}0.65 \\
(1)\end{array}$ & 0.42 \\
\hline Being a good mother is a reward in itself. & $\begin{array}{l}\text { Agree } \\
\text { Disagree }\end{array}$ & $\begin{array}{c}177 \\
1\end{array}$ & $\begin{array}{l}70 \\
36\end{array}$ & $\begin{array}{l}28.3 \\
97.3\end{array}$ & $\begin{array}{l}65.41 \\
(1)\end{array}$ & $<0.001$ \\
\hline
\end{tabular}




\section{DISCUSSION}

In this study a total of 284 puerperal women were enrolled. The mean age (in years) \pm SD was $27.39 \pm$ 5.26. The minimum age was 18 years and maximum age was 45 years. According to cut-off score $\geq 13$ on Edinburg postnatal depression scale $37.3 \%$ women were found depressed while $62.7 \%$ women were found non-depressed. The mean EPDS score \pm SD was $10.12 \pm 6.27$ ( $\min$ score 0 ; $\max$ score 27 ). The mean EPDS scores in a similar study conducted by Demiroz et al were $12 \pm 4.7$. The prevalence of postnatal depression was found as $47.3 \%$ [11]. Eckerdal et al in their study found the prevalence of postnatal depression as $13 \%$ in their study and Gebregziabher et al found prevalence of postnatal depression as $7.4 \%$ [12, 13].

Women's age, women's education level, household head's education level, family monthly income and socioeconomic status are closely related to postpartum depression in the current study, whereas the occupation of head of family had no significant association with postnatal depression among puerperal women. Akin to findings of this study, the higher age of women was found as a important analyst of postnatal depression in a study carried out by Smorti et al [14]. The education of women was also associated with postnatal depression in a study conducted by Dadi et al, Matsumura et al, Chien et al, and Huang et al [15-18]. However, Roomruangwong and Smorti et al [14, 19] could not establish a significant relationship of women's education with postnatal depression in their studies. The low family income and economic status was found as significant predictor of postnatal depression in studies carried out by Gebregziabher et al, Dadi et al, Chien et al and Huang et al, $[13,15,17,18]$ whereas, Ozmen et al reported no significance of family income with postnatal depression [20]. Similar to the findings of this study, various studies reported no association of occupation with postnatal depression [19] [14, 20].

In this study, delivery method, delivery location, number of pregnancies, history of infant deaths, history of child deaths, history of miscarriage and the number of living children were significantly associated with postnatal depression, however, the contraception had no significant effect on postnatal depression. Erkerdal et al and Malik et al found in their study that women who had cesarean section were found as having more risk of developing postnatal depression as compared to women who had normal vaginal delivery $[12,21]$. The history of miscarriage, infant death and child death was not found significantly associated with postnatal depression in a study carried out by Eastwood et al [22]. The study of Mathisen et al testified that the women who had two or more children were found as more depressed compared to women who had less children [23]. The contraception failure and unplanned pregnancy was considerably associated with postnatal depression in the study carried out by Gebregziabher et al [13]. Smorti et al and Roomruangwong found no significant association of contraception or planned pregnancy with postnatal depression [14, 19].

\section{CONCLUSION}

Socio-demographic, obstetric risk factors, and parenting sense of competence are significant predictors of postnatal depression that need to be addressed in order to sustain safe motherhood.

\section{REFERENCES}

1. Alzahrani AD. Risk Factors for Postnatal Depression among Primipara Mothers. Span J Psychol. 2019 Jul 12;22:E35.

2. Al Dallal FH, Grant IN. Postnatal depression among Bahraini women: prevalence of symptoms and psychosocial risk factors. East Mediterr Health J. 2012;18(5):439-45.

3. Halbreich U, Karkun S. Cross-cultural and social diversity of prevalence of postpartum depression and depressive symptoms. Journal of affective disorders. 2006;91(2-3):97-111.

4. Klainin P, Arthur DG. Postpartum depression in Asian cultures: a literature review. International journal of nursing studies. 2009;46(10):1355-73.

5. Ghaedrahmati M, Kazemi A, Kheirabadi G, Ebrahimi A, Bahrami M. Postpartum depression risk factors: A narrative review. Journal of education and health promotion. 2017;6.

6. Slomian J, Honvo G, Emonts P, Reginster J-Y, Bruyère $O$. Consequences of maternal postpartum depression: A systematic review of maternal and infant outcomes. Women's Health. 2019;15:1745506519844044.

7. De Oliveira CVR, Rasheed M, Yousafzai AK. Chronic Maternal Depressive Symptoms Are Associated With Reduced Socio-Emotional Development in Children at 2 Years of Age: Analysis of Data From an Intervention Cohort in 
Rural Pakistan. Frontiers in Psychiatry. [Original Research]. 2019 2019-November-20;10(859).

8. Shrestha SD, Pradhan R, Tran TD, Gualano RC, Fisher JR. Reliability and validity of the Edinburgh Postnatal Depression Scale (EPDS) for detecting perinatal common mental disorders (PCMDs) among women in low-and lower-middle-income countries: a systematic review. BMC Pregnancy Childbirth. 2016 Apr 4;16:72.

9. Dolatian M, Maziar P, Majd HA, Yazdjerdi M. The relationship between mode of delivery and postpartum depression. Journal of Reproduction \& Infertility. 2006;7(3).

10. Cox JL, Holden JM, Sagovsky R. Detection of postnatal depression: development of the 10-item Edinburgh Postnatal Depression Scale. The British Journal of Psychiatry. 1987;150(6):782-6.

11. Demiröz HP, Taştan K. The effects of perceived social support on postpartum depression. J Surg Med. 2018;2(3):298-302.

12. Eckerdal P, Georgakis MK, Kollia N, Wikström A$\mathrm{K}$, Högberg U, Skalkidou A. Delineating the association between mode of delivery and postpartum depression symptoms: a longitudinal study. Acta Obstetricia et Gynecologica Scandinavica. 2018;97(3):301-11.

13. Gebregziabher NK, Netsereab TB, Fessaha YG, Alaza FA, Ghebrehiwet NK, Sium AH. Prevalence and associated factors of postpartum depression among postpartum mothers in central region, Eritrea: a health facility based survey. BMC Public Health. 2020;20(1):1614.

14. Smorti M, Ponti L, Pancetti F. A Comprehensive Analysis of Post-partum Depression Risk Factors: The Role of Socio-Demographic, Individual, Relational, and Delivery Characteristics. Frontiers in Public Health. [Original Research]. 2019 2019October-24;7(295).

15. Dadi AF, Miller ER, Mwanri L. Postnatal depression and its association with adverse infant health outcomes in low- and middle-income countries: a systematic review and meta-analysis. BMC pregnancy and childbirth. 2020;20(1):416.
16. Matsumura K, Hamazaki K, Tsuchida A, Kasamatsu $\mathrm{H}$, Inadera $\mathrm{H}$. Education level and risk of postpartum depression: results from the Japan Environment and Children's Study (JECS). BMC Psychiatry. 2019;19(1):419.

17. Chien L-Y, Tai C-J, Yeh M-C. Domestic decisionmaking power, social support, and postpartum depression symptoms among immigrant and native women in Taiwan. Nursing Research. 2012;61(2):103-10.

18. Huang $T$, Rifas-Shiman $S L$, Ertel $K A$, Rich-Edwards J, Kleinman K, Gillman MW, et al. Pregnancy hyperglycaemia and risk of prenatal and postpartum depressive symptoms. Paediatric and perinatal epidemiology. 2015;29(4):281-9.

19. Roomruangwong $C$, Withayavanitchai $S$, Maes $M$. Antenatal and postnatal risk factors of postpartum depression symptoms in Thai women: A casecontrol study. Sexual \& Reproductive Healthcare. 2016;10:25-31.

20. Ozmen D, Cetinkaya AC, Ulas SC, Ozmen E. Association between perceived social support and postpartum depression in Turkey. Journal of Advances in Medicine and Medical Research. 2014:2025-36.

21. Malik F, Malik B, Irfan M. Comparison of postnatal depression in women following normal vaginal delivery and caesarean section: A pilot study. Journal of Postgraduate Medical Institute. 2015 01/01;29:34-7.

22. Eastwood JG, Jalaludin BB, Kemp LA, Phung HN, Barnett BEW. Relationship of postnatal depressive symptoms to infant temperament, maternal expectations, social support and other potential risk factors: findings from a large Australian crosssectional study. BMC pregnancy and childbirth. 2012 2012/12/12;12(1):148.

23. Mathisen SE, Glavin K, Lien L, Lagerløv P. Prevalence and risk factors for postpartum depressive symptoms in Argentina: a crosssectional study. International journal of women's health. 2013;5:787.

\footnotetext{
This is an Open Access article distributed under the terms of the Creative Commons Attribution License (http://creativecommons.org/licenses/by/4.0), which permits unrestricted use, distribution, and reproduction in any medium, provided the original work is properly cited.
} 